Rev Biomed 2002; 13:15-23.

\title{
Valor diagnóstico de anticuerpos \\ anti Helicobacter pylori en pacientes referidos al Servicio de Endoscopia Digestiva del Hospital San Vicente de Paul, Costa Rica.
}

\section{Artículo Original}

Eugenia M. Quintana-Guzmán ${ }_{1}^{1}$, Pilar Salas-Chaves ${ }^{2}$, Rosario Achí-Araya ${ }^{2}$, Henry Davidovich-Rose ${ }^{3}$, Karl Schosinsky-Nevermann .

${ }^{1}$ Departamento de Análisis Clínicos, Facultad de Microbiología, Instituto de Investigaciones en Salud (INISA), Universidad de Costa Rica, ${ }^{3}$ Coordinador del Servicio de Endoscopía Digestiva, Hospital San Vicente de Paul, Heredia, Costa Rica.

\section{RESUMEN.}

Introducción. El estudio se realizó en 78 pacientes referidos al Servicio de Endoscopía Digestiva del Hospital San Vicente de Paul, un estudio de anticuerpos séricos anti $H$. pylori tipo $\operatorname{IgG}, \operatorname{IgM}$ e IgA por el método de ELISA utilizando antígenos comerciales y preparados con bacterias autóctonas, a fin de determinar la sensibilidad diagnóstica y la especificidad diagnóstica de cada inmunoglobulina. Material y métodos. Se utilizó un método de ELISA comercial y dos métodos de ELISA preparados en el laboratorio utilizando antígenos no comerciales, uno de origen costarricense y otro preparado en Suecia.

Resultados. El 83\% de los pacientes presentaron infección por H. pylori. En la determinación de IgG anti $H$. pylori el método de ELISA comercial presentó la sensibilidad diagnóstica más alta (76\%) mientras que el método de ELISA no comercial con antígeno de origen costarricense presentó la sensibiliodad diagnóstica más baja (57\%), pero con la mayor especificidad diagnóstica (92\%). El método de ELISA no comercial más específico fue el que utiliza antígeno de origen costarricense, sin embargo el antígeno de origen sueco mostró mayor sensibilidad (67\%).

Conclusiones. El uso de antígenos autóctonos permite detectar la presencia de $H$. pylori con mayor confianza que los foráneos, ya que una prueba serológica aumentada es altamente sugestiva de la presencia de la bacteria en la mucosa gástrica. La inmunoglobulina anti $H$. pylori con mejor sensibilidad y especificidad diagnóstica fue la IgG, pero se recomienda realizar un estudio

Solicitud de sobretiros: MSc. Eugenia Ma. Quintana-Guzmán, Departamento de Análisis Clínicos, Facultad de Microbiología, Universidad de Costa Rica, San Pedro, San José, Costa Rica. Tel: 207-4388/207-5440 Fax: 2075440/225-2374/231-17-46 E-mail: euquin@cariari.ucr.ac.cr Recibido el 4/Enero/2001. Aceptado para publicación el 19/Febrero/2001. 
EM Quintana-Guzmán, P Salas-Chaves, R Achí-Araya, H Davidovich-Rose y col.

histológico de biopsia gástrica para un adecuado diagnóstico de la presencia de $H$. pylori en la mucosa gástrica, ya que éste no se debe hacer utilizando únicamente el estudio serológico.

(Rev Biomed 2002; 13:15-23)

Palabras clave: $H$. pylori, seroprevalencia, ELISA, Costa Rica.

\section{SUMMARY.}

Diagnostic value of Helicobacter pylory antibodies in patients referred to the digestive endoscopy service at the Hospital San Vicente de Paul, Costa Rica.

Introduction. Antibodies titers against $H$. pylori antigens were determined in 78 patients referred to the Digestive Endoscopy Service of the San Vicente de Paul Hospital, Costa Rica. The assay was performed by enzyme immunoassay (ELISA), using commercial and non comercial methods with costarrican bacterial antigens and antigens prepared in Sweden, with the purpose of determining the diagnostic sensitivity and specificity of $\operatorname{IgG}, \operatorname{IgM}$ and IgA inmunoglobulines.

Material and methods. The commercial ELISA method was performed according to instructions and the two ELISA methods were prepared in the laboratory using no commercial antigens, one from Costa Rica and the other prepared in Sweden.

Results. H. pylori infection was present in $83 \%$ of studied patients. The determination of $\mathrm{IgG}$ anti H. pylori by commercial ELISA method presents the highest diagnostic sensitivity (76\%) and the non commercial ELISA using costarrican antigen shows the lowest diagnostic sensitivity (57\%) with the highest diagnostic specificity $(92 \%)$. The non commercial ELISA method more specific was the one that uses costarrican bacterial antigen, but the antigen prepared in Sweden had the highest sensitivity $(67 \%)$.

Discussion. The use of antigens prepared of local strains permits the detection of the presence of $H$. pylori with greater confidence as compared to antigens prepared with strains from other regions, because elevated serologic tests are high suggestive of the presence of the bacteria in the gastric mucous. The anti $H$. pylori inmunoglobulin with the highest diagnostic sensitivity and specificity was the IgG, but a histologic study of gastric biopsy instead of using the serologic test alone is recommended for the diagnosis of $H$. pylori.

(Rev Biomed 2002; 13:15-23)

Key words: $H$. pylori, seroprevalence, ELISA, Costa Rica.

\section{INTRODUCCION.}

La bacteria Helicobacter pylori tiene gran importancia médica debido a su implicación en la patología gastroduodenal. (1). Ha sido reconocida como el agente causal más importante de úlcera duodenal (2) y úlcera gástrica (3), y tiene un papel importante en la patogénesis del cáncer gástrico (4).

El diagnóstico de gastritis asociada a H. pylori se basa en diferentes técnicas que varían en su grado de agresividad, eficacia y disponibilidad en términos de costo-beneficio. Dentro de estas técnicas se logra el diagnóstico mediante la determinación de la respuesta del huésped a la infección por medio de anticuerpos anti $H$. pylori en suero, saliva, jugo gástrico o cultivos de mucosa duodenal, pruebas de ureasa o por observación microscópica de cortes histológicos de biopsia gástrica $(5,6)$.

La infección por $H$. pylori induce a una respuesta inmune local y sistémica. La respuesta inmune a $H$. pylori al nivel de la mucosa es predominantemente de tipo $\operatorname{IgA}(7)$ mientras que la sistémica es esencialmente a inmunoglobulinas de la clase IgG (8).

La detección de la respuesta sistémica del tipo IgA no ha sido un hallazgo importante y los títulos de anticuerpos a un conjunto de antígenos de células completas de $H$. pylori se han demostrado más altos para la clase $\operatorname{IgG}$ que para la $\operatorname{IgA}(8,9)$.

\section{Revista Biomédica}


Anticuerpos contra H. pylori en Costa Rica.

Los anticuerpos IgM no difieren entre individuos H. pylori positivos y los negativos (9).

Se han desarrollado varios métodos serológicos para la detección de H. pylori (10). Las pruebas serológicas constituyen un medio sencillo y poco invasivo para la detección de esta infección. Existen diferentes métodos aplicables al diagnóstico serológico incluyendo aglutinación bacteriana, fijación de complemento, hemaglutinación pasiva, inmunofluorescencia indirecta, aglutinación mediante partículas de látex marcadas con antígeno bacteriano, inmunoelectrotransferencia y técnicas de enzimoinmunoanálisis, siendo el método de ELISA el más sensible y el más utilizado (11).

La técnica de ELISA es un método diagnóstico sencillo y rápido que posibilita la realización de estudios epidemiológicos con la ventaja de poder obtener resultados cuantitativos, lo que permite establecer distintos valores de positividad para diferentes grupos poblacionales y evaluar la respuesta al tratamiento (12).

El aislamiento, la identificación y la purificación de antígenos específicos ha llevado al desarrollo de varios sistemas de ELISA, algunos de los cuales han sido incorporados a juegos de reactivos comerciales disponibles $(13,14)$.

En las diversas técnicas de ELISA desarrolladas para la detección de anticuerpos específicos frente a $H$. pylori se han empleado diferentes preparaciones antigénicas incluyendo organismos vivos, bacterias tratadas con formalina, sonicados bacterianos y tratados con calor, ultracentrifugado de sonicados bacterianos, antígenos termoestables, extractos antigénicos con glicerina ácida, preparaciones de ureasa bacteriana, antígeno de citotoxina, proteínas celulares de alto peso molecular y proteínas de membrana externa (15).

En esta investigación nos propusimos realizar un estudio de anticuerpos en suero anti $H$. pylori tipo IgG, IgM e IgA por el método de ELISA a fin de determinar la sensibilidad y especificidad diagnóstica para cada inmunoglobulina. Para ello se utilizó un juego de reactivos de una casa comercial (DPC Biermann GmbH) y dos métodos preparados en el laboratorio utilizando antígenos no comerciales obtenidos de bacterias $H$. pylori autóctonas y de bacterias de pacientes suecos.

\section{MATERIAL Y METODOS.}

Muestras: se obtuvo muestras de biopsia gástrica antral y de suero con material estéril descartable de 78 pacientes referidos al Servicio de Gastroscopía del Hospital San Vicente de Paul, Heredia, Costa Rica. Todos los pacientes incluidos en el estudio dieron su consentimiento para participar en la investigación que contó con la aprobación del Comité de Ética del Hospital. A cada paciente se le realizó análisis histológico de biopsias de antro gástrico, frotis por aposición teñido con Giemsa, pruebas rápidas de ureasa y cultivo. Se consideraron positivas por $H$. pylori las biopsias que mostraron resultados positivos con cualquiera de estos métodos y negativas las que no mostraron positividad con ninguna de las pruebas. Además, a cada paciente se le realizó estudio de anticuerpos anti $H$. pylori por ensayo inmunoenzimático utilizando un método de ELISA comercial en 62 muestras para IgG y dos métodos de ELISA preparados en el laboratorio utilizando antígenos no comerciales para $\operatorname{IgG}, \operatorname{IgM}$ e $\operatorname{Ig} \mathrm{A}$ en los 78 pacientes.

Estudio histológico: el estudio histológico de las biopsias gástricas se llevó a cabo en el Servicio de Patología del Hospital México usando la tinción de hematoxilina - eosina y azul de toludina.

Frotis por aposición teñido con Giemsa: se realizaron frotis por aposición de las biopsias gástricas en portaobjetos fijándose con alcohol metílico por 3 minutos. Luego se cubrió con el colorante de Giemsa (29) y se dejó a temperatura ambiente de 15 a 30 minutos para observar microscópicamente la presencia de bacilos característicos en el frotis. 
EM Quintana-Guzmán, P Salas-Chaves, R Achí-Araya, H Davidovich-Rose y col.

\section{Pruebas de ureasa:}

Rojo fenol: la preparación de los reactivos se llevó a cabo de acuerdo con Arvid y col. (30). Cada biopsia fue colocada en un volumen de 0,2 $\mathrm{ml}$ de reactivo y mantenida a temperatura ambiente por un minuto. Se utilizó un blanco de reactivos como control negativo. Una prueba positiva se presenta cuando ocurre cambio de color de amarillo a rojo antes de los 20 minutos de incubación.

O-cresolftaleína complexona (OCPC): se preparó una solución de trabajo conteniendo EDTA $2,5 \mathrm{mmol} / \mathrm{L}$, urea $100 \mathrm{mg} / \mathrm{dl}$ y OCPC $2,5 \mathrm{mmol} / \mathrm{L}$ a $\mathrm{pH}$ 7,0. Cada biopsia fue colocada en un volumen de $0,5 \mathrm{ml}$ de reactivo e incubada a temperatura ambiente. Las lecturas se realizaron al minuto, a los 30, 60, 90 y 120 minutos utilizando el reactivo de OCPC como control negativo. Una prueba positiva se presenta cuando aparece un color púrpura (31).

Cultivo: las biopsias gástricas se transportaron en medio Cary-Blair y posteriormente fueron inoculadas en agar sangre con TTC al 1\% (agar sangre Columbia con sangre de caballo desfibrinada) para ser incubadas de 5 a 7 días en jarra de anaerobiosis sin catalítico con sobre de Gas-Pack a $37^{\circ} \mathrm{C}$. A todas las colonias se les realizó tinción de Gram y a las bacterias bacilos Gram negativos oxidasa positivos se les realizó la prueba de ureasa, catalasa y movilidad . Enayos inmunoenzimáticos:

ELISA comercial: se utilizó un juego de reactivos de $\operatorname{IgG} H$. pylori Milenia de la DPC Biermann GmbH (32) según las recomendaciones de la casa comercial.

ELISA con antígeno de pacientes costarricenses: se utilizó como antígeno una suspención de bacterias totales, cultivadas de biopsia gástrica de pacientes costarricenses, en 0,5 ml PBS-Tween con $0,01 \% \mathrm{NaN}_{3}$ la cual fue sonicada y determinada su concentración proteíca en $500 \mathrm{mg} / \mathrm{dL}$. Las placas de microtiter fueron recubiertas con $5 \mathrm{~g} /$ pozo del antígeno durante 1824 horas a temperatura ambiente. Se adicionó 100 $\mu \mathrm{L}$ de suero fetal bovino por 2 horas para bloquear

\section{Revista Biomédica}

los espacios no saturados. Se utilizó inmunoglobulinas de conejo anti humanas IgA, IgG e IgM marcadas con fosfatasa alcalina. Al día siguiente se agregó $100 \mu \mathrm{L}$ del sustrato de paranitrofenol $1 \mathrm{mg} / \mathrm{mL}$ en amortiguador de dietanolamina $1 \mathrm{M}$ pH 9.8. Los títulos de anticuerpos anti $H$. pylori fueron expresados como títulos relativos, el valor de densidad óptica a 405 $\mathrm{nm}$ a los 60 minutos de agregado el sustrato multiplicado por el factor de dilución de 1000. El punto de corte fue determinado obteniendo el promedio más dos desviaciones estándard de los títulos relativos obtenidos en los pacientessin tratamiento por $H$. pylori que presentaron tanto estudio histológico como cultivo, tinción de Giemsa y pruebas de ureasa negativas.

ELISA con antígeno preparado en Suecia: como antígeno se utilizó el sobrenadante de un sonicado de bacterias de la cepa control de $H$. pylori NCTC-11637 y cuatro aislamientos No. 128, 132, 134 y 151, preparado en el Laboratorio de Bacteriología Oral, Instituto Karolinska, Hospital Huddinge, Suecia. Las placas de microtiter fueron recubiertas con $100 \mu \mathrm{L} /$ pozo del antígeno y se procedió igual al método anterior.

\section{RESULTADOS.}

Por el método obtenido de la casa comercial se analizaron los niveles séricos de $\operatorname{IgG}$ a 62 pacientes, mientras que con los métodos preparados en el laboratorio se analizaron 78

\section{Cuadro 1}

Estudio serológico de 62 sueros para IgG anti $\boldsymbol{H}$. pylori por diferentes métodos de ELISA.

\begin{tabular}{|c|c|c|c|c|}
\hline \multirow{2}{*}{$\begin{array}{l}\text { Método } \\
\text { ELISA }\end{array}$} & \multicolumn{2}{|c|}{ No. de muestras } & \multicolumn{2}{|c|}{ No. de falsos } \\
\hline & Positivas & Negativas & Positivo & Negativo \\
\hline Comercial & 40 & 22 & 5 & 16 \\
\hline ELISA 1 & 16 & 46 & 3 & 38 \\
\hline ELISA 2 & 31 & 31 & 5 & 25 \\
\hline
\end{tabular}

1: antígeno de origen costarricense

2: antígeno de origen sueco 
Anticuerpos contra H. pylori en Costa Rica.

Cuadro 2

Sensibilidad y especificidad diagnóstica de los métodos de ELISA en la determinación de IgG anti $H$. pylori.

\begin{tabular}{lcc}
\hline Método & Sensibilidad diagnóstica (\%) & Especificidad diagnóstica(\%) \\
\hline ELISA comercial & 76 & 79 \\
ELISA 1 & 57 & 92 \\
ELISA 2 & 67 & 78 \\
\hline
\end{tabular}

1: antígeno de origen costarricense

2: antígeno de origen sueco

Sensibilidad diagnóstica: verdaderos positivos verdaderos positivos + falsos negativos

Especificidad diagnóstica: verdaderos negativos

verdaderos negativos + falsos positivos

muestras de suero por IgG, IgM e IgA.

En la muestra analizada se encontró un $83 \%$ de pacientes positivos verdaderos por $H$. pylori y un $17 \%$ de pacientes negativos verdaderos por esta bacteria.

En los cuadros No. 1, 2, 3 y 4 se muestran los resultados obtenidos en este estudio. Cabe mencionar que dos pacientes recibieron tratamiento previo contra la infección por $H$. pylori, por lo tanto no se consideraron al calcular la especificidad diagnóstica.

\section{DISCUSION.}

La serología ha encontrado su principal aplicación en los estudios epidemiológicos y se ha utilizado para conocer los porcentajes de prevalencia de $H$. pylori en varias poblaciones e investigar factores como el origen geográfico, la situación socioeconómica, los antecedentes étnicos y la edad (5).

Otra importante indicación de las investigaciones serológicas es su aplicación en el seguimiento del tratamiento de erradicación a largo plazo. Varios estudios han demostrado que el descenso significativo en el título de anticuerpos IgG e IgA generalmente se produce después de seis meses de una erradicación satisfactoria (20).

Actualmente es posible la determinación de clases independientes de inmunoglobulinas séricas (IgG, IgA, IgM, IgE) específicas frente a H. pylori. La inmunoglobulina predominante entre estas clases de anticuerpos séricos circulantes es la IgG. En la literatura se ha reportado con respecto a IgG en suero una sensibilidad del 81 al $100 \%$ y una especificidad entre 73 y $100 \%$ con un valor

Cuadro 3

Estudio serológico de 78 sueros para inmunoglobulinas anti $H$. pylori por ELISA.

\begin{tabular}{|c|c|c|c|c|c|c|c|c|c|c|c|c|}
\hline & \multicolumn{6}{|c|}{ No. de muestras } & \multicolumn{6}{|c|}{ No. de falsos } \\
\hline & \multicolumn{3}{|c|}{$\operatorname{IgG} \frac{\text { Positivas }}{\operatorname{Ig} M \operatorname{Ig} A}$} & \multicolumn{3}{|c|}{$\frac{\text { Negativas }}{\operatorname{IgG} \operatorname{IgM} \operatorname{Ig} \mathrm{A}}$} & \multicolumn{3}{|c|}{$\frac{\text { Positivos }}{\text { IgG IgM IgA }}$} & \multicolumn{3}{|c|}{$\frac{\text { Negativos }}{\operatorname{IgG} \operatorname{IgM} \operatorname{Ig} A}$} \\
\hline ELISA1 & 22 & 10 & 27 & 56 & 66 & 51 & 3 & 2 & 4 & 45 & 54 & 41 \\
\hline ELISA 2 & 37 & 22 & 36 & 38 & 56 & 43 & 4 & 3 & 5 & 30 & 46 & 36 \\
\hline
\end{tabular}

1: antígeno de origen costarricense

2: antígeno de origen sueco 
EM Quintana-Guzmán, P Salas-Chaves, R Achí-Araya, H Davidovich-Rose y col.

Cuadro 4

Sensibilidad y especificidad diagnósticas de ELISA en la determinación de inmunoglobulinas anti $H$. pylori.

\begin{tabular}{cccccccc}
\hline & \multicolumn{3}{c}{ Sensibilidad diagnóstica (\%) } & & \multicolumn{3}{c}{ Especificidad diagnóstica (\%) } \\
\cline { 3 - 6 } & $\operatorname{IgG}$ & $\operatorname{IgM}$ & $\operatorname{IgA}$ & & $\operatorname{IgG}$ & $\operatorname{IgM}$ & $\operatorname{IgA}$ \\
\hline ELISA 1 & 53 & 49 & 55 & 92 & 85 & 85 \\
ELISA 2 & 63 & 52 & 59 & 85 & 78 & 78 \\
\hline
\end{tabular}

1: antígeno de origen costarricense

2: antígeno de origen sueco

predictivo de infección entre el 90 y 95\% (5).

Las respuestas de IgG en personas infectadas involucran a las cuatro subclases de inmunoglobulina $\mathrm{G}\left(\mathrm{IgG}_{1}, \mathrm{IgG}_{2}, \mathrm{IgG}_{3}\right.$ e $\left.\mathrm{IgG}_{4}\right)$, pero parece ser predominante de las subclases $\operatorname{IgG}_{1}$ e $\operatorname{IgG}_{2} \circ \operatorname{IgG}_{1}, \operatorname{IgG}_{2}$ e $\operatorname{IgG}_{4}$. El estudio de los niveles de subclases independientes de IgG no resulta superior a la detección del nivel de $\operatorname{IgG}$ total para conseguir el diagnóstico serológico de la infección por H. pylori (5).

La inmunoglobulina IgA específica frente a $H$. pylori constituye el principal anticuerpo implicado en la respuesta inmune a nivel local. La respuesta sistémica suele ser menos marcada que la de $\mathrm{IgG}$, sin embargo sus niveles en suero parecen indicar un grado más severo de inflamación de la mucosa. Aunque también es posible la determinación de IgM específica frente a $H$. pylori su utilidad diagnóstica parece ser limitada ya que se detecta raramente en individuos infectados (5). Sin embargo en este estudio se observa una sensibilidad y especificidad clínica semejantes a IgA.

De nuestro estudio podemos concluir que el $83 \%$ de los pacientes estudiados presentaron infección por $H$. pylori y que en el caso de la determinación de $\mathrm{IgG}$ anti $H$. pylori el método de ELISA comercial presentó la sensibilidad diagnóstica más alta (76\%) con una especificidad de $79 \%$ mientras que el método de ELISA no comercial con antígeno de origen costarricense presentó la sensibilidad diagnóstica más baja (57\%) pero con la especificidad diagnóstica más alta (92\%). Esto nos indica que el método que mostró menor número de muestras falsas positivas fue el ELISA no comercial con antígeno costarricense, pero que a su vez fue el que más falsos negativos presentó.

A pesar de que en el ELISA comercial se usaron cepas de $H$. pylori ajenas a nuestro medio, los resultados de sensibilidad clínica fueron muy buenos en comparación con los otros métodos no comerciales, es decir, fueron los que presentaron menor número de falsos negativos, posiblemente por el mejor desempeño analítico del método comercial.

El método de ELISA no comercial más específico fue el que utiliza antígeno de origen costarricense, sin embargo el preparado en Suecia mostró una mayor sensibilidad. Por lo tanto no encontramos ninguna ventaja metodológica en cuanto a sensibilidad clínica, al utilizar antígenos producto de las bacterias aisladas de pacientes costarricenses. Sin embargo, si se desea disminuir el número de falsos positivos, se recomienda el uso de antígenos autóctonos. Un valor superior al establecido (valor de corte mayor) es altamente sugestivo de que el paciente presenta H. pylori en mucosa gástrica. Posiblemente esto se deba a una menor interferencia debido a la disminución de reacciones cruzadas.

En cuanto a las inmunoglobulinas IgG, IgM e IgA analizadas por métodos no comerciales, 
Anticuerpos contra H. pylori en Costa Rica.

podemos concluir que la inmunoglobulina anti $H$. pylori con mejor sensibilidad y especificidad diagnóstica es sin duda la IgG, como lo han indicado otros investigadores $(5,8,9)$. Esto por cuanto la IgG anti $H$. pylori fue la inmunoglobulina que presentó el menor número de falsos positivos y falsos negativos, tanto con el antígeno de origen costarricense como con el preparado en Suecia.

De los falsos negativos por el método de ELISA se podría suponer que se trata de pacientes recién infectados o que presentan poca respuesta inmunológica hacia $H$. pylori. Otra posibilidad se puede deber a que la población costarricense presenta títulos elevados para $H$. pylori lo que haría que el valor de corte sea alto aún para los pacientes verdaderos negativos, elevándose el número de falsos negativos. La presencia de falsos positivos podría deberse a reacciones cruzadas o al efecto de parche en el análisis por biopsia debido a la distribución desigual del $H$. pylori en la mucosa gástrica $(21,22)$. Aún más, la disminución de la especificidad diagnóstica de las pruebas serológicas también puede deberse a que con frecuencia se prescriben antibióticos para infecciones comunes como las respiratorias superiores e infecciones del tracto urinario que como en el caso del metronidazol y claritromicina, erradican al $H$. pylori en un 17-20\% de los pacientes (23). Esto hace que estas pruebas serológicas se mantengan positivas mientras que la actividad de la ureasa es negativa y no se observa esta bacteria en el estudio histológico (24). Esta situación puede resultar en una prueba serológica falsa positiva.

Otra condición que podría disminuir la especificidad de las pruebas serológicas es la presencia de agentes que alteren la mucosa gástrica produciendo inflamación, distribución desigual bacteriana y alteración de la actividad de la ureasa $(25,26)$. Estos agentes pueden ser compuestos de bismuto, inhibidores de la bomba de protones y los mismos antibióticos, que dificultan la detección histológica de $H$. pylori y aumentan la probabilidad de falsos positivos en las pruebas serológicas.

Con objeto de que la técnica sea lo más sensible y específica posible, el antígeno usado debe contener componentes compartidos por la mayoría de cepas de $H$. pylori sin mostrar reacciones cruzadas con otros antígenos bacterianos. La introducción de antígenos más purificados reduce la aparición de reacciones cruzadas y mejora la especificidad de la técnica, lo que también se logra mediante el uso de antígenos autóctonos.

A pesar de la alta especificidad aportada por estos antígenos parcial o altamente purificados su empleo individualizado puede conllevar algunas desventajas principalmente debidas a la reducción del nivel de sensibilidad del método (5).

La composición del antígeno ideal todavía no ha sido lograda, sin embargo parece razonable pensar que la combinación de dos o más antígenos purificados podría considerarse adecuada (5).

Si bien, la aplicación de la serología presenta pocas ventajas al diagnóstico, aporta conocimiento al nivel epidemiológico de la infección por $H$. pylori y posiblemente sea útil para seguimiento del tratamiento a largo plazo. La interpretación de los resultados obtenidos mediante procedimientos serológicos debe ser cautelosa y siempre respaldada por la historia clínica del paciente. Se recomienda realizar un estudio histológico de biopsia gástrica para un adecuado diagnóstico de la presencia de la bacteria en la mucosa gástrica (27), y no hacerlo utilizando únicamente el estudio serológico.

\section{AGRADECIMIENTO.}

Al Departamento de Bacteriología Clínica del Instituto Karolinska, Suecia, por facilitarnos antígeno de H. pylori así como placas de microtítulo utilizadas en los ELISAS no comerciales.

\section{REFERENCIAS.}

1.- Grimley C, Holder R, Loft D, Morris A, Nwokolo C. Helicobacter pylori-associated antibodies in patients with duodenal ulcer, gastric and oesophageal adenocarcinoma. Eur J Gastroenterol Hepatol 1999; 11: 503-9.

2.- Velhuyzen S, Sherman Ph. Helicobacter pylori infection as a cause of gastritis, duodenal ulcer, gastric cancer and non ulcer dyspepsia: a systematic overview. Can 


\section{EM Quintana-Guzmán, P Salas-Chaves, R Achí-Araya, H Davidovich-Rose y col.}

Med Assoc J 1994; 150: 177-85.

3.- Labenz J, Borsch G. Evidence for the essential role of Helicobacter pylori in gastric ulcer disease. Gut 1994; 35: 19-22.

4.- Correa P. Helicobacter pylori and gastric carcinogenesis. Am J Sur Phatol 1995; 19 (suppl I): 53743.

5.- Sanz J, López-Brea M. Diagnóstico serológico de la infección por Helicobacter pylori. En Lopez-Brea M. Helicobacter pylori. Microbiología, clínica y tratamiento. s.ed; Madrid: Mosby/Doyma Libros; 1995. p. 153-174.

6.- Christie J, McNulty C, Shepherd N, Valori R. Is saliva serology useful for diagnosis of Helicobacter pylori? Gut 1996; 39: 27-30.

7.- Hayashi S, Sugiyama T, Misano K, Awakawa T, Kurokawa I, Yachi A, et al. Quantitative detection of secretory immunoglobin A to Helicobacter pylori in gastric juice : antibody capture enzyme linked immunosorbent assay. J Clin Lab Anal 1996; 10: 74-7.

8.- Rathbone B, Wyatt J, Worsley B. Systemic and local antibody responses to gastric Campylobacter pyloridis in nonulcer dispepsia. Gut 1986; 27: 642-7.

9.- Perez-Perez G, Dworkin B, Chodos J, Blaser M. Campylobacter pylori antibodies in humans. Ann Intern Med 1988; 109:11-7.

10.- Simor A, Lin E, Saibil F, Cohen L, Louie M, Pearen $\mathrm{S}$, et al. Evaluation of enzyme immunoassay for detection of salivary antibody to Helicobacter pylori. J Clin Microbiol 1996; 34: 550-3.

11.- Newell D. The principles and practices of the serodiagnosis of Helicobacter pylori infection. Lab Medica International 1991; VIII (6): 7-11.

12.- López-Brea M, Martín E, Alarcón T, Acuña M, Gimeno M, Sanz J. Seguimiento de la respuesta serológica cuantitativa al tratamiento de la infección por Helicobacter pylori en niños. Enf Infecc Microbiol Clin 1993; 11: 33-5.

13.- Gossens H, Glupczynski Y, Burette A, van den Borre C, Butzler J. Evaluation of a comercially available second-generation in inmunoglobulin $\mathrm{G}$ enzyme immunoassay for detection of Helicobacter pylori infection. J Clin Microbiol 1995; 30:179-80.

\section{Revista Biomédica}

14.- Xia H, Kalantar J, Wyatt J, Adams S, Cheung K, Eslick G, et al. High sensitivity and specificity of a laboratory-based serological test, pylori Dtect ELISA, for detection of Helicobacter pylori infection. Diag Microbiol Infect Dis 2000; 36: 69-74.

15.- Partipilo M, Woster P. The role of Helicobacter pylori in peptic ulcer disease. Pharmacotherapy 1993; 13: $330-9$.

16.- Sáenz G. Hematología Analítica. 3era ed: San José: EDNASSS; 1995. p. 109.

17.- Arvid A, Cook R, Tabaqchali S, Farthing M. One minute endoscopy room test for Campylobacter pylori. Lancet 1988; 1:704.

18.- Quintana E, Schosinsky K, Arias M, Davidovich H. Comparative srudy of urease tests for Helicobacter pylori detection in gastric biopsies. Rev Biomed 1999; 10:14551.

19.- Milenia IgG H. pylori. DPC Biermann GmbH. Diagnostic Product Corporation. PIMKHP-C.

20.- Taylor D, Eaton M, Chang N and Salama S. Construction of a Helicobacter pylori Genome Map and Demonstration of Diversity at the Genome Level. J Bacteriol 1992; 174: 6800-6.

21.- Azuma T, Kato T, Hirai M, Ito S, Kohli Y. Diagnosis of Helicobacter pylori infection. J Gastroenterol Hepatol 1996, 11: 662-9.

22.- Van der Hulst R, Verheul S, Weel J, Gerrits Y, tenKate F, Dankert J. Effects of specimen collection techniques, transport media, and incubation of cultives on the detection rate of Helicobacter pylori . Eur J Clin Microbiol Infect Dis 1996; 15:211-5.

23.- Chiba N, Rao B, Rademaker,J, Hunt R. Meta-analysis of the efficacy of antibiotic therapy in eradicating Helicobacter pylori. Am J Gastroenterol 1992; 87: 171627.

24.- Peterson W, Graham D, Marshal B, Blaser M, Genta $\mathrm{R}$, Klein P. Clarithromicin as monotherapy for eradication of Helicobacter pylori: a randomized, double blind trial. Am J Gastroenterol 1993; 88: 1860-4.

25.- Chey W, Fey D, Scheiman J, Nostrant T, Delvalle J. The role of acid suppression in the effects of lansoprazole 
and ranitidine on the ${ }^{14} \mathrm{C}$ urea breath test. Gastroenterol 1996; 110:80.

26.- Logan R, Walker M, Misiewicz J, Gummett P, Karim $\mathrm{Q}$, Baron J. Changes in the intragastric distribution of Helicobacter pylori during treatment with omeprazole. Gut 1995; 36:12-6.

27.- Quintana E, Schosinsky K, Davidovich H, Taylor L, Arias M. Inmunoglobulina $G$ anti Helicobacter pylori por ELISA y Western-blot en pacientes del Servcio de Gastroenterología del Hospital San Vicente de Paúl, Heredia. Acta Med Cost 2000; 42:19-24. 